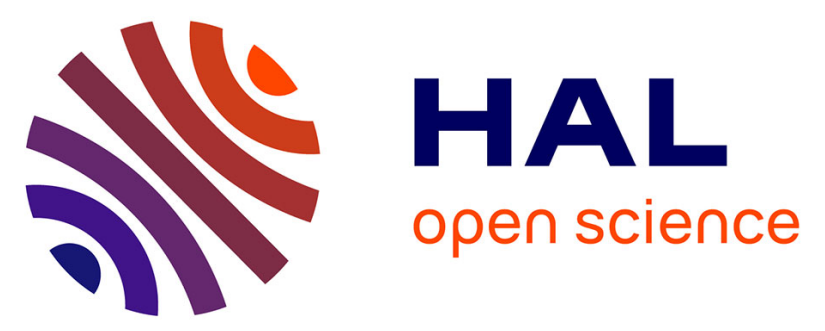

\title{
Photon-excitation photon-emission maps (PhexPhem maps) with rovibronic resolution as a data base for theory and astrophysics part I: method and first results for $\mathrm{H} 2$
}

\author{
Philipp Schmidt, André Knie, Andreas Hans, Kouichi Hosaka, Masatoshi \\ Ukai, Michèle Glass-Maujean, Arno Ehresmann
}

\section{To cite this version:}

Philipp Schmidt, André Knie, Andreas Hans, Kouichi Hosaka, Masatoshi Ukai, et al.. Photonexcitation photon-emission maps (PhexPhem maps) with rovibronic resolution as a data base for theory and astrophysics part I: method and first results for H2. Journal of Physics B: Atomic, Molecular and Optical Physics, 2021, 54 (3), pp.034001. 10.1088/1361-6455/abcfda . hal-03146322

\section{HAL Id: hal-03146322 \\ https://hal.sorbonne-universite.fr/hal-03146322}

Submitted on 19 Feb 2021

HAL is a multi-disciplinary open access archive for the deposit and dissemination of scientific research documents, whether they are published or not. The documents may come from teaching and research institutions in France or abroad, or from public or private research centers.
L'archive ouverte pluridisciplinaire HAL, est destinée au dépôt et à la diffusion de documents scientifiques de niveau recherche, publiés ou non, émanant des établissements d'enseignement et de recherche français ou étrangers, des laboratoires publics ou privés. 
PAPER • OPEN ACCESS

Photon-excitation photon-emission maps (PhexPhem maps) with rovibronic resolution as a data base for theory and astrophysics part I: method and first results for $\mathrm{H}_{2}$

To cite this article: Philipp Schmidt et al 2021 J. Phys. B: At. Mol. Opt. Phys. 54034001

View the article online for updates and enhancements.

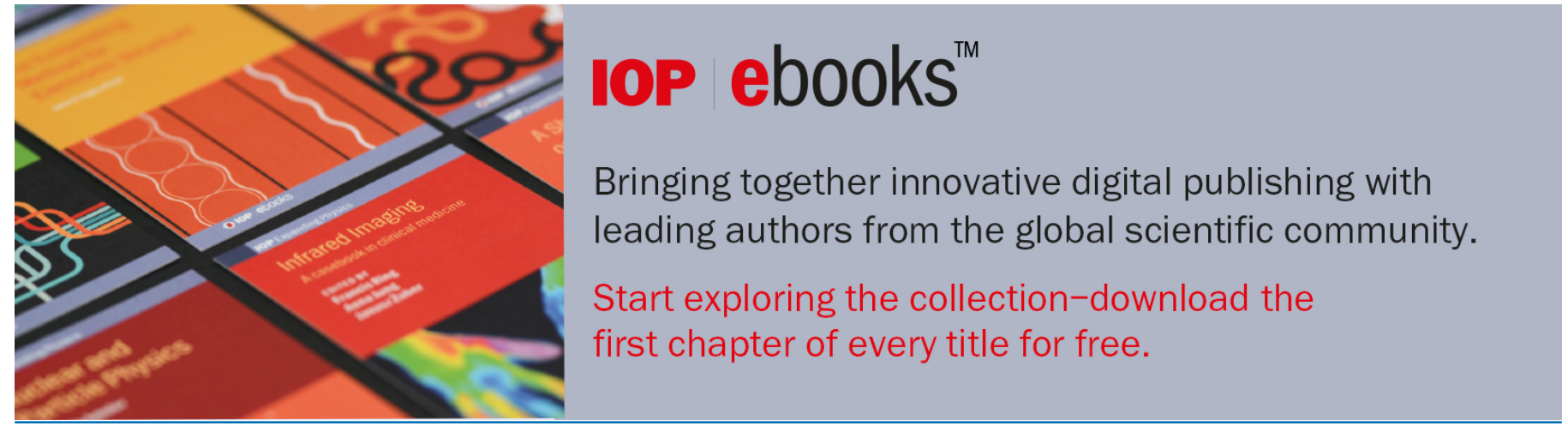

This content was downloaded from IP address 212.198.232.121 on 19/02/2021 at 07:02 


\title{
Photon-excitation photon-emission maps (PhexPhem maps) with rovibronic resolution as a data base for theory and astrophysics part I: method and first results for $\mathrm{H}_{2}$
}

\author{
Philipp Schmidt ${ }^{1}$, Andre Knie ${ }^{1} \odot$, Andreas Hans ${ }^{1}{ }^{\circledR}$, Kouichi Hosaka ${ }^{2}$, \\ Masatoshi Ukai ${ }^{3}$, Michele Glass-Maujean ${ }^{4}$ and Arno Ehresmann ${ }^{1, *} \mathbb{}(1)$ \\ ${ }^{1}$ Institute of Physics and Center for Interdisciplinary Nanostructure Science and Technology, University \\ of Kassel, Heinrich-Plett-Str. 40, D-34132 Kassel, Germany \\ 2 Department of Chemistry, Tokyo Institute of Technology, Meguro-ku, Tokyo 152-8551, Japan \\ ${ }^{3}$ Department of Applied Physics, Tokyo University of Agriculture and Technology, Koganei-shi, \\ Tokyo-184-8588, Japan \\ ${ }^{4}$ Laboratoire d'Etudes du Rayonnement et de la Matière en Astrophysique et Atmosphères, UMR-8112, \\ Sorbonne Université, Faculté des Sciences et Ingénierie, 4 pl Jussieu, F-75005 Paris, France
}

E-mail: ehresmann@physik.uni-kassel.de

Received 15 August 2020, revised 19 November 2020

Accepted for publication 2 December 2020

Published 20 January 2021

\begin{abstract}
Dispersed fluorescence of wavelengths between 75 and $180 \mathrm{~nm}$ has been recorded after excitation of room temperature $\mathrm{H}_{2} X^{1} \Sigma_{g}^{+}(\mathrm{v}=0)$ by narrow bandwidth monochromatized synchrotron radiation. Spectra have been taken in the exciting-photon energy range between 11 and $18 \mathrm{eV}$ for discrete energy steps of $2.5 \mathrm{meV}$ at a bandwidth of about $10 \mathrm{meV}$. The exciting-photon energy bandwidth has been narrow enough to induce transitions between individual rovibronic states. The resolution in the dispersed fluorescence detection was partly sufficient to resolve fluorescent transitions between individual rovibronic states. The recorded intensities are presented as two-dimensional photon-excitation photon-emission (PhexPhem) maps. They give a complete overview of experimental dispersed VUV/ FUV fluorescence spectra of $\mathrm{H}_{2}$ for these excitation energies, covering excitation from $\mathrm{H}_{2} X^{1} \Sigma_{g}^{+}(\mathrm{v}=0)$ to all known singly excited states. The presented proof of principle experiments show a way to generate spectroscopic reference data for theory benchmarking and the interpretation of astrophysical observations.
\end{abstract}

Keywords: $\mathrm{H}_{2}$, photon emission, fluorescence, singly excited states, photon excitation

(Some figures may appear in colour only in the online journal)

* Author to whom any correspondence should be addressed.

(c) (i) Original content from this work may be used under the terms of the Creative Commons Attribution 4.0 licence. Any further distribution of this work must maintain attribution to the author(s) and the title of the work, journal citation and DOI.

\section{Introduction}

Molecular physics and physical chemistry is unthinkable without the interplay between the development of experimental methods to investigate and quantify molecular properties and the development of corresponding theoretical methods and 
models. The hydrogen molecule, as nature's simplest neutral molecule, has ever been the benchmark for such advancements in fundamental molecular physics and it goes without saying that $\mathrm{H}_{2}$ is the prototype molecule to teach molecular physics.

$\mathrm{H}_{2}$ is also a useful probe for astrophysical processes (Wakelam et al 2017) as it is the most abundant molecule in space. Therefore, quantitative data on its photon absorption and photon emission spectrum are of highest importance. $\mathrm{H}_{2}$ is a homonuclear molecule and so transitions between rovibrational levels of the same electronic potential are dipoleforbidden, causing no (or very weak quadrupolar) features in the infrared spectral region. Consequently, the major absorption or emission features are caused by transitions between rovibronic levels of the electronic ground state and those of the singly-excited states, lying in the vacuum ultraviolet (VUV) or far ultraviolet (FUV) spectral range. Photons or absorption features from such processes emitted by astrophysical objects cannot be observed on Earth as its atmosphere is opaque in this spectral range, except the absorbing or emitting $\mathrm{H}_{2}$ cloud possesses a redshift, shifting the VUV/FUV features into the visible spectral range. With the advent of rocket and satellite based observations, however, this obstacle has been removed. $\mathrm{H}_{2}$ in space was identified by Carruthers (1970) by a rocketborne spectrometer, absorption lines were then observed by the Copernicus satellite telescope (Spitzer et al 1974, Morton and Dinerstein 1976) and its VUV-absorption spectrum was observed by the FUV spectroscopic explorer (Moos et al 2000) and the Hubble Space Telescope (Meyer et al 2001). A recent example for $\mathrm{H}_{2}$ being a probe for fundamental processes is the discussion whether the electron to proton mass ratio was different in the early Universe or in strong gravitational fields. Here line shifts of rovibronic $\mathrm{H}_{2}$ transitions observed in space with respect to highly accurate laboratory data may be used to verify such a difference (Salumbides et al 2015, Ubachs et al 2016, Ubachs 2018, Ubachs et al 2019 and references therein).

Quantitative laboratory VUV/FUV absorption and emission spectra of $\mathrm{H}_{2}$ may also be used to interpret modifications in astrophysical spectra recorded by Earth or satellite bound detection systems due to absorption and-potentially_reemission of photons along the line of sight. Corresponding consequences have to be considered when trying to correlate the recorded Earth- or satellite bound data to processes in space. Again, due to its abundance $\mathrm{H}_{2}$ is a major cause for such spectral modifications. Here, apart from the precise knowledge of energy differences between rovibronic levels, the quantitative knowledge of energy-resolved photon absorption and emission probabilities is, therefore, important to interpret such spectra.

For $\mathrm{H}_{2}$ the list of scientific work published on particular details of its complex spectrum is too long for being cited here completely, some advanced theoretical models are described by Glass-Maujean et al (2013), Staszewska and Wolniewicz (2002), and Abgrall et al (1993). While each of these studies possesses its particular value an experimental record of all possible dipole-allowed excitations of the lowest lying singly excited $\mathrm{H}_{2}$ states and their subsequent spectrally resolved emission in one experiment is as yet missing. Recently, Ubachs et al (2019) presented a list of experimentally determined highly accurate absorption line energies along with calculated oscillator strengths and damping parameters of the $\mathrm{H}_{2}$ Lyman and Werner band systems and Xiong et al (2019) presented experimentally determined oscillator strengths with an extensive comparison to preceding work. What is missing to date, however, are quantitative experimentally determined intensities of spectrally dispersed emission lines after excitation by small-bandwidth photons on a joint intensity scale. In the current work, we describe the method how to obtain such highlyresolved dispersed photon emission spectra as a function of the exciting-photon energy. In the experimental data below we will plot photon emission intensities as a function of both, exciting-photon energy and photon emission wavelength in order to better distinguish excitation and emission. We will focus on the photon emission wavelength range between 70 and $180 \mathrm{~nm}$ after excitation by narrow bandwidth synchrotron radiation between 11 and $18 \mathrm{eV}$. The excitation energy range covers excitation from room temperature populated rotational levels of $\mathrm{H}_{2} X^{1} \Sigma_{g}^{+}(\mathrm{v}=0)$ to all known singly excited states with a bandwidth narrow enough to excite transitions between individual rovibronic levels. Emitted photons are recorded in a wavelength range covering the complete VUV/FUV spectral range emitted by transitions between rovibronic levels of the singly excited states to discrete levels and the continuum of the ground state, including emission from excited atomic dissociation fragments. The data is displayed as a twodimensional photon-excitation photon-emission map (henceforth called PhexPhem map), where intensities are plotted as a function of both, the emitted-photon wavelength (or energy) and the exciting-photon energy. In a PhexPhem map molecular processes like dipole-allowed rovibronic transitions between bound states, transitions between a bound state and a dissociation continuum, predissociation, and ionization will be visible at the same time. The current paper describes proof of concept experiments where it is shown that it is possible to record dispersed photon emission in the full excitation-emission range relevant to excitation and emission of all singly excited $\mathrm{H}_{2}$ states and to display such spectra in one image. A quantitative intensity determination will be the task of future experiments.

In the first section the experiments and the representation of the data as a PhexPhem map is described in greater detail. It follows a discussion of its main features by a comparison to simulations. The presented simulations do not have the ambition, to advance the description of a specific molecular physics problem, rather than to correlate specific molecular processes to signatures in the PhexPhem map. Although the main features are all known to some detail from past work the PhexPhem map representation of the spectra has its value on its own, as it simplifies considerably the orientation in the complex $\mathrm{H}_{2}$ emission spectrum.

\section{Experimental}

\subsection{Set up overview}

The set-up to map the exciting-photon energy dependent dispersed VUV/FUV emission spectra of gaseous species after excitation by monochromatized synchrotron radiation is based 


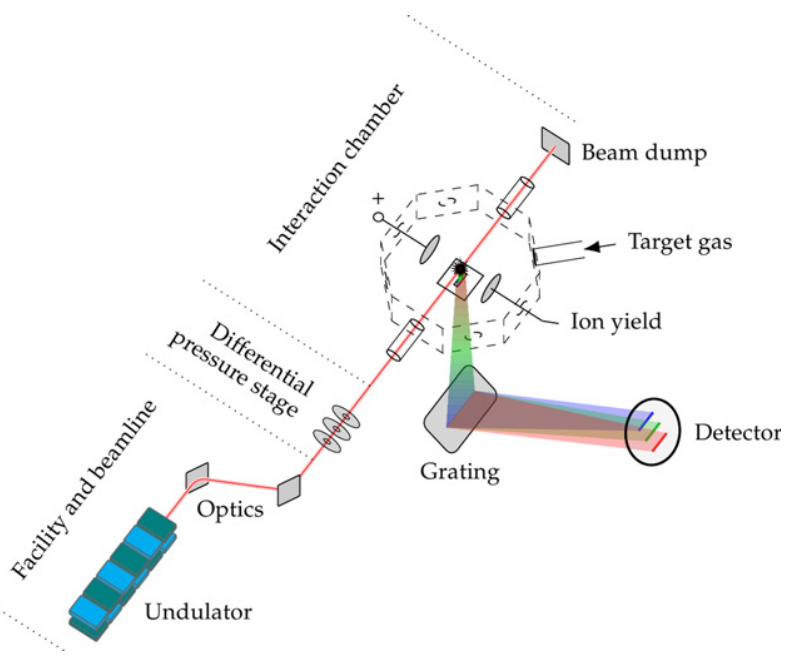

Figure 1. Sketch of the experimental set-up to determine the PhexPhem maps. Synchrotron radiation from the U125/2 undulator of BESSY II, Helmholtz-Zentrum Berlin is dispersed by the $10 \mathrm{~m}$ normal incidence monochromator (10 mNIM). The differential pressure stage between beamline and interaction chamber and the interaction chamber itself are home-built. Photon emission is recorded at $90^{\circ}$ with respect to the polarization axis of the synchrotron radiation by a $1 \mathrm{~m}$ NIM spectrometer, alternatingly equipped with two types of position sensitive detection systems (see text).

on a set of experimental techniques collectively called photoninduced fluorescence spectroscopy (PIFS) (Schmoranzer et al 1986, Schmoranzer et al 2001, Zimmermann et al 2004, Hans et al 2018) which in several cases has also been used to determine absolute emission cross sections (Ehresmann et al 1994a,1994b). A sketch of the current set-up is displayed in figure 1. Briefly, synchrotron radiation from an undulator source is monochromatized by a primary monochromator and focussed into a target cell. Photons emitted from the target gas at room temperature are dispersed by a secondary spectrometer and recorded by a position sensitive detection system. We will now describe the individual components of this experiment one by one.

\subsection{Undulator and beamline}

Exciting-photons have been delivered by the U125/2 10 m-NIM beamline of BESSYII, Helmholtz-Zentrum Berlin, which supplies narrow-bandwidth monochromatized photons in the energy range between 5 and $40 \mathrm{eV}$ (corresponding to exciting-photon wavelengths between 250 and $30 \mathrm{~nm}$ ). The U125/2 undulator consists of 32 periods quasi-periodically arranged $125 \mathrm{~mm}$ wide permanent magnets to suppress higher harmonics (Bahrdt et al 2001). Its planar design restricts the polarization to linear horizontal. The beamline is equipped with a 10 m normal-incidence off-Rowland circle monochromator (Reichardt et al 2001). This beamline achieves a resolution of up to $E / \Delta E=85000$ using a grating of 1200 lines/mm in 2nd order and $10 \mu \mathrm{m}$ slit sizes (Baumgaertel $\mathrm{P}$ and Packe I 2016). For the current experiments an exit slit width of $500 \mu \mathrm{m}$ has been chosen resulting in a bandwidth of $10 \mathrm{meV}$. This bandwidth allowed the individual excitation of rovibronic transitions in absorption from the ground state of molecular hydrogen (figure 2). The photon energy range provided by this instrument allows access to the valence region of many atomic and molecular systems. For molecular hydrogen and its isotopes it covers the excitation energy range of molecular singly and doubly excited states completely. For the current experiment the excitation of the singly excited states is addressed. Therefore dispersed photon-emission spectra are recorded for exciting-photon energies between 11 and $18 \mathrm{eV}$ in individual exciting-photon energy steps of $2.5 \mathrm{meV}$ for spectra recorded in the VUV photon emission range and of $5 \mathrm{meV}$ in the FUV photon emission range. The larger steps in the FUV where due to a compromise between available beamtime and the task of covering the whole excitation energy range for the singly excited states. The linearity between the nominally addressed exciting-photon energy at the beamline monochromator control and the true energy of the exciting-photons has been checked by comparing known absorption energies of transitions between rovibronic levels of the $\mathrm{H}_{2}$ ground state and those of singly excited states (Herzberg and Jungen 1972, Takezawa 1970, Glass-Maujean et al 2013 and references therein) to energies of peaks in the (undispersed) fluorescence excitation function. Figure 2 shows that the nominal and the true photon energies of the monochromatized synchrotron radiation are agreeing well and that individual rovibronic transitions can be identified.

\subsection{Interaction chamber}

In the differentially pumped interaction chamber (GlassMaujean et al 2010a, 2013, 2017, Hans et al 2015) a pressure of typically 20 mTorr has been maintained using a magnetically operated gas valve controlled by a capacitance manometer. Additionally two electrodes are placed perpendicular to the incoming synchrotron radiation, one on positive potential, the other grounded, to measure a signal proportional to the ion yield. At the downstream end of the interaction chamber, a Faraday cup measures the amount of synchrotron radiation transmitted through the target cell. This value is used together with the photocurrent of the last refocusing mirror of the beamline to determine the incoming photon flux $I_{0}$.

\subsection{Spectrometer and detectors}

The emitted photons are dispersed by a $1 \mathrm{~m}$ normal-incidence McPherson type 225 spectrometer. The fluorescence wavelength range between 75 and $180 \mathrm{~nm}$ has been recorded by two different spectrometer-grating detector combinations. In the spectral range below $130 \mathrm{~nm}$ (from now on called vacuum ultraviolet (VUV) range) a ruled Au coated spherical grating of $12001 \mathrm{~mm}^{-1}$ has been used, blazed at $80 \mathrm{~nm}$, in combination with an open-face micro channel plate (MCP) stack in front of a wedge-and-stripe anode for position detection. In the spectral range between 115 and $180 \mathrm{~nm}$ (from now on called far ultraviolet (FUV) spectral range) the spectrometer has been equipped with an Al coated spherical grating blazed at $150 \mathrm{~nm}$ combined with a sealed position-sensitive detector consisting of a combination of a CsTe photocathode on an $\mathrm{MgF}_{2}$ window and an MCP stack for signal amplification with a delay-line anode for position detection. The detectors are single-particle 


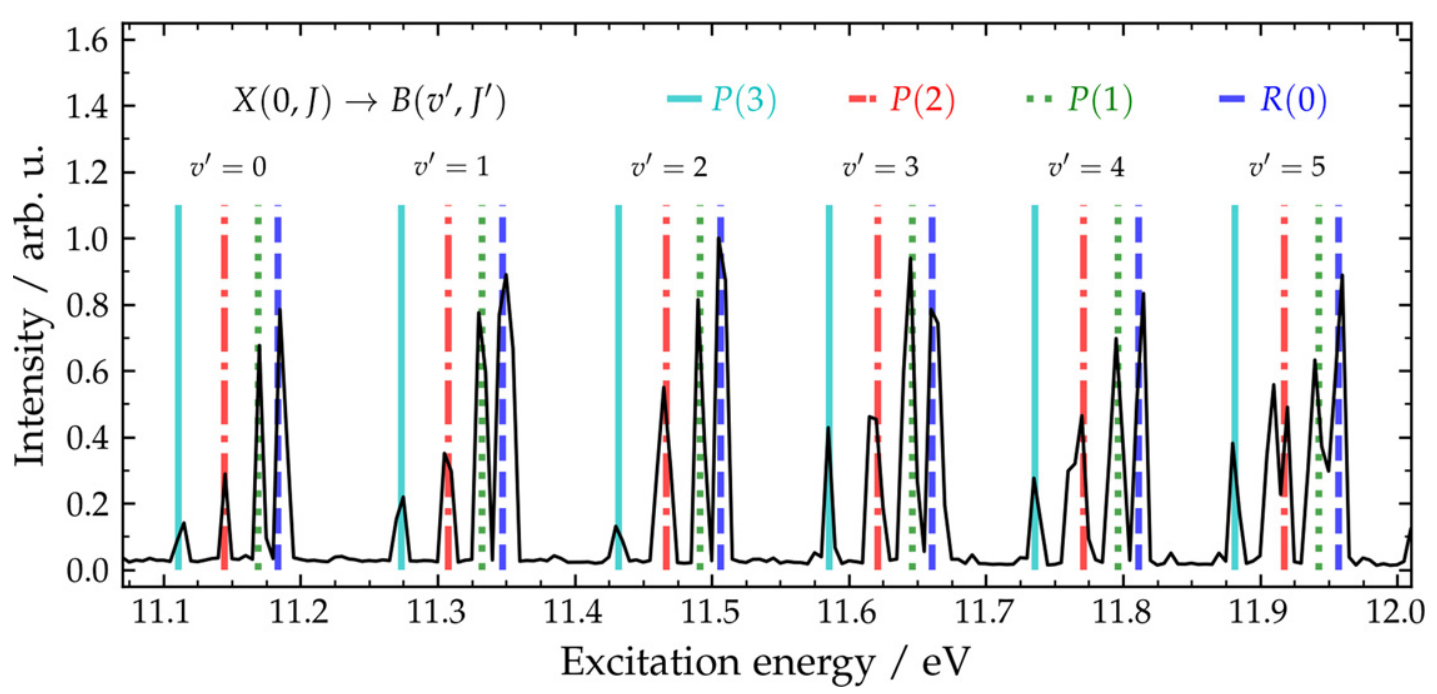

Figure 2. Exemplary (undispersed) fluorescence excitation function obtained by plotting the fluorescence-wavelength integrated dispersed intensities in the recorded spectra as a function of the exciting-photon energy used for exciting-photon energy calibration. Assignments are restricted to selected calculated energies (see text) of rovibronic levels of the B state to prove rotational resolution in excitation and linearity in the exciting-photon energy scale.

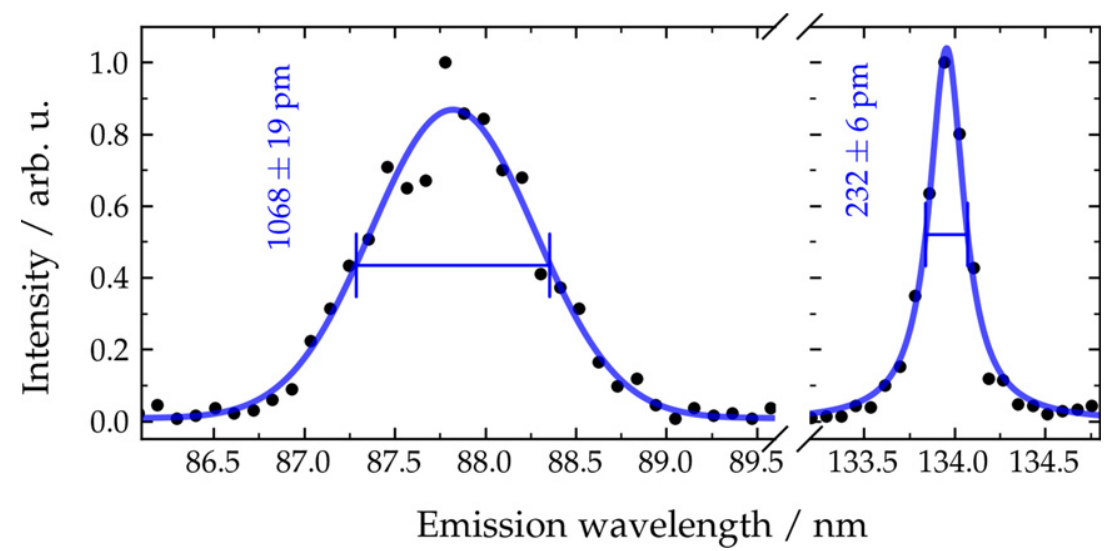

Figure 3. Examples for the determination of the resolution of the used spectrometer grating/ photon detector combinations in the VUV (left) and FUV (right) spectral ranges. The emission lines are molecular band transitions with natural linewidths in the range of several fm and a Doppler broadening of $<1 \mathrm{pm}$.

counting (Hans et al 2018) devices. These amplify one-photon photoionisation events on a cathode surface, resulting in higher signal-to-noise ratios for very-low intensity gas-phase experiments at synchrotron radiation facilities with high repetition rates when compared to charge-coupled devices. Spectra have been taken at first inner order with a spectrometer entrance slit of $500 \mu \mathrm{m}$ close to the interaction region. With such a setting typically a spectral range of $30 \mathrm{~nm}$ at a time can be recorded on a $50 \mathrm{~mm}$ active detector surface. The recorded linewidths are determined by the full widths at half maximum (FWHM) of the spectrometer-detector combination as the Doppler as well as the natural line widths for the current experiment are well below the FWHM determined by the apparatus. The FWHM has been determined for the two grating-detector combinations in separate experiments, where a prototypical result is displayed in figure 3 . For the two spectral ranges the resolving powers have been

$$
\frac{\lambda}{\Delta \lambda_{\mathrm{VUV}}} \approx 80, \quad \frac{\lambda}{\Delta \lambda_{\mathrm{FUV}}} \approx 570
$$

with $\Delta \lambda$ equaling the FWHM determined as described above. The FUV detector had a significantly higher resolution as compared to the VUV one. A rotational resolution in emission could therefore only be achieved in the FUV spectral region.

\subsection{Determination of line intensities}

The spectrum at a specific excitation energy $E_{\text {exc }}$ displayed on a position sensitive detector at a specific spectrometer-grating angle $g a$ is a matrix of signal values $S_{g a}\left(E_{\mathrm{exc}}, x_{D}, y_{D}\right)$ at positions $\left(x_{D}, y_{D}\right)$ of the detector surface. Positions $\left(x_{D}, y_{D}\right)$ on the detector surface have been digitized into $512 \times 512$ pixels for the present experiment to make efficient use of the 


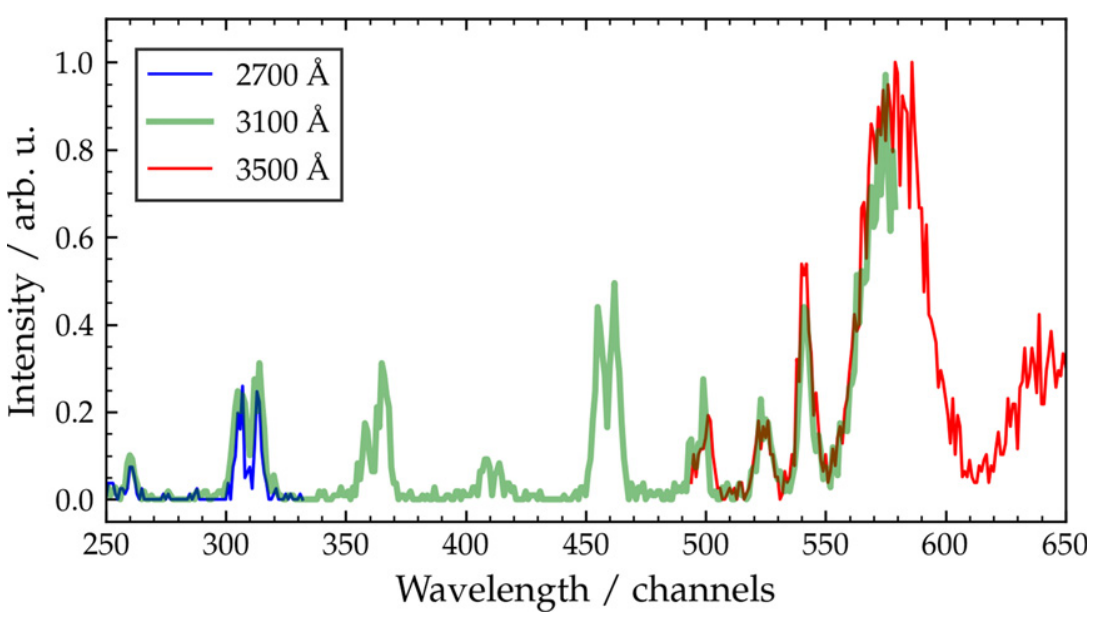

Figure 4. Example how three raw spectra (blue, green, red) taken at different grating angles are appended at the same exciting-photon energy. The grating angles nicknamed by their nominal central wavelengths. This procedure has been carried out for all spectra taken at the different grating angles at each exciting-photon energy. The wavelength axis has not yet been calibrated.

resolution of the analog-to-digital conversion. The spectrum displays dispersed images of the columnar source volume. Line intensities have been determined by integrating pixels belonging to one spectral line along the axis perpendicular to the dispersion plane $S_{g a}\left(E_{\text {exc }}, x_{D}\right)=\int_{y_{D}} S_{g a}\left(E_{\text {exc }}, x_{D}, y_{D}\right) \mathrm{d} y_{D}$. The conversion function of detector position $x_{D}$ versus emission wavelength $\lambda$ has also been determined by least squares fitting of wavelengths of known emission features (e.g. Abgrall et al (1993): Lyman and Werner bands) to detector positions. The resulting linear functions were the same for all spectra taken in the FUV for the different spectrometer grating angles and the same for all VUV spectra, but different between the two spectral ranges. Both conversion functions resulted in an unequivocal assignment of the observed spectral features to transitions. Finally, the recorded signals have been subtracted by the background signal $B_{k}$ and normalized for the measurement time $t$, the target density $\rho$, the incoming photon flux $I_{0}$, and for varying quantum efficiency $Q(\lambda)$ of the spectrometer-grating detection systems to result in an intensity as a function of emission wavelength (for one detector image digitized in 512 channels):

$$
I_{g a}\left(E_{\mathrm{exc}}, \lambda\right)=\frac{\left(S_{g a}\left(E_{\mathrm{exc}}, \lambda\right)-B_{k}\right)}{t \cdot \rho \cdot I_{0} \cdot Q(\lambda)} .
$$

One spectrum has been obtained for each energy step $E_{\text {exc }}$ across the exciting-photon energy range. As the necessary fluorescence spectral range (here between 75 and $180 \mathrm{~nm}$ ) cannot be covered by one specific angle $g a$ of the spectrometer grating (where a spectral range of $30 \mathrm{~nm}$ is covered by one angle), spectra of several grating angles have been recorded for each $E_{\text {exc }}$ with large spectral overlaps. The intensities integrated over the overlap spectral range for each exciting-photon energy have been used to append the spectra of different $g a$. An example how this has been done is shown in figure 4 for three different $g a$. To cover the full range of $E_{\text {exc }}$ in $2.5 \mathrm{meV}$ steps 2801 exciting-photon energy steps were necessary with 8 spectra taken at each $E_{\text {exc }}$ to cover the full fluorescence wavelength range resulting in more than 20000 spectra.
The full data set is shown as a two-dimensional color-scale PhexPhem intensity map as a function of both the excitingphoton energy (horizontal axis) and the emission wavelength (vertical axis). The intensity scale in such a representation has to be chosen carefully in order to visualize both, features due to small intensity variations and very strong spectral features at the same time. For this purpose we used the colormap viridis introduced by van der Walt and Smith into the MatPlotLib library of Python. Still, the dynamic range between very intense emissions (e.g. the Lyman- $\alpha$ line of atomic hydrogen fragments) and the majority of features is too large for a joint presentation. The intensities $I\left(E_{\mathrm{exc}}, \lambda\right)$ have, therefore, been scaled by a non-linear transformation. We used a bound exponential scaling function with a single parameter $a$. The relative intensity $F=\frac{I}{I_{\max }}$ determined from the measured intensities $I$ and $I_{\max }$ is scaled to the displayed relative intensity values $F_{d}=\frac{I_{d}}{I_{\max , d}}$ by the transformation:

$$
F_{d}=1-e^{-a F}
$$

The effect of this transformation is shown in figure 5(a) for different $a$. The two-dimensional PhexPhem map of $\mathrm{H}_{2}$ has been displayed in this work with $a=15$ after reducing the intensity of the strongest spectral feature (the $\mathrm{Ly}_{\alpha}$-line) by a factor of 10 .

The complete PhexPhem map is finally obtained by joining the spectra of the two different spectral ranges. Here the transition between the different emission-wavelength calibrations in the FUV and VUV must be considered. Due to the available calibration points, the two intercepts of the calibration curves coincide well in the overlap region of the two spectral ranges as shown in figure 5(b). A completed result for the two-dimensional VUV/FUV PhexPhem map of $\mathrm{H}_{2}$ is shown in figure 6. The underlying data represents a set of photon excitation emission intensities of $\mathrm{H}_{2}$ covering the complete exciting-photon energy range from room-temperature populated rovibronic levels of the vibronic ground state to the singly excited molecular states and the subsequent complete dispersed emission in the VUV/FUV spectral range on a joint 
(a)

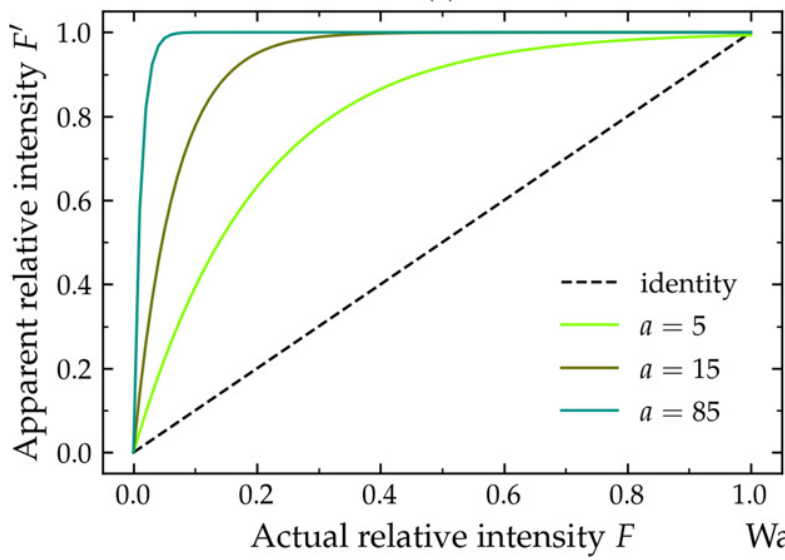

(b)

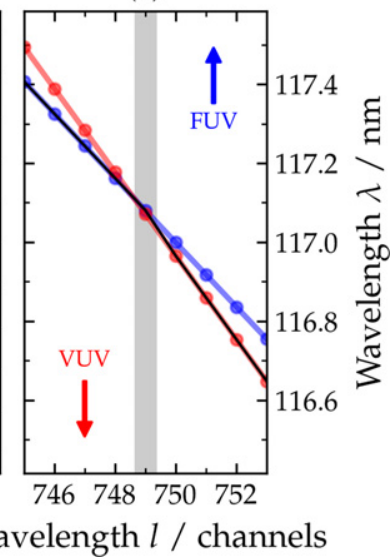

Figure 5. (a) The non-linear scaling applied to display the PhexPhem maps for different parameters $a$. It projects the dynamic range into a small interval at lower intensities to increase their contrast at the cost of reduced details for intense emissions. (b) Procedure to append the different wavelength calibrations of the VUV (red) and FUV (blue) spectral ranges for a joint plot. The gray bar represents the channel offset between the two spectral regions. The resulting wavelength calibration for the composed map is added as a black line.

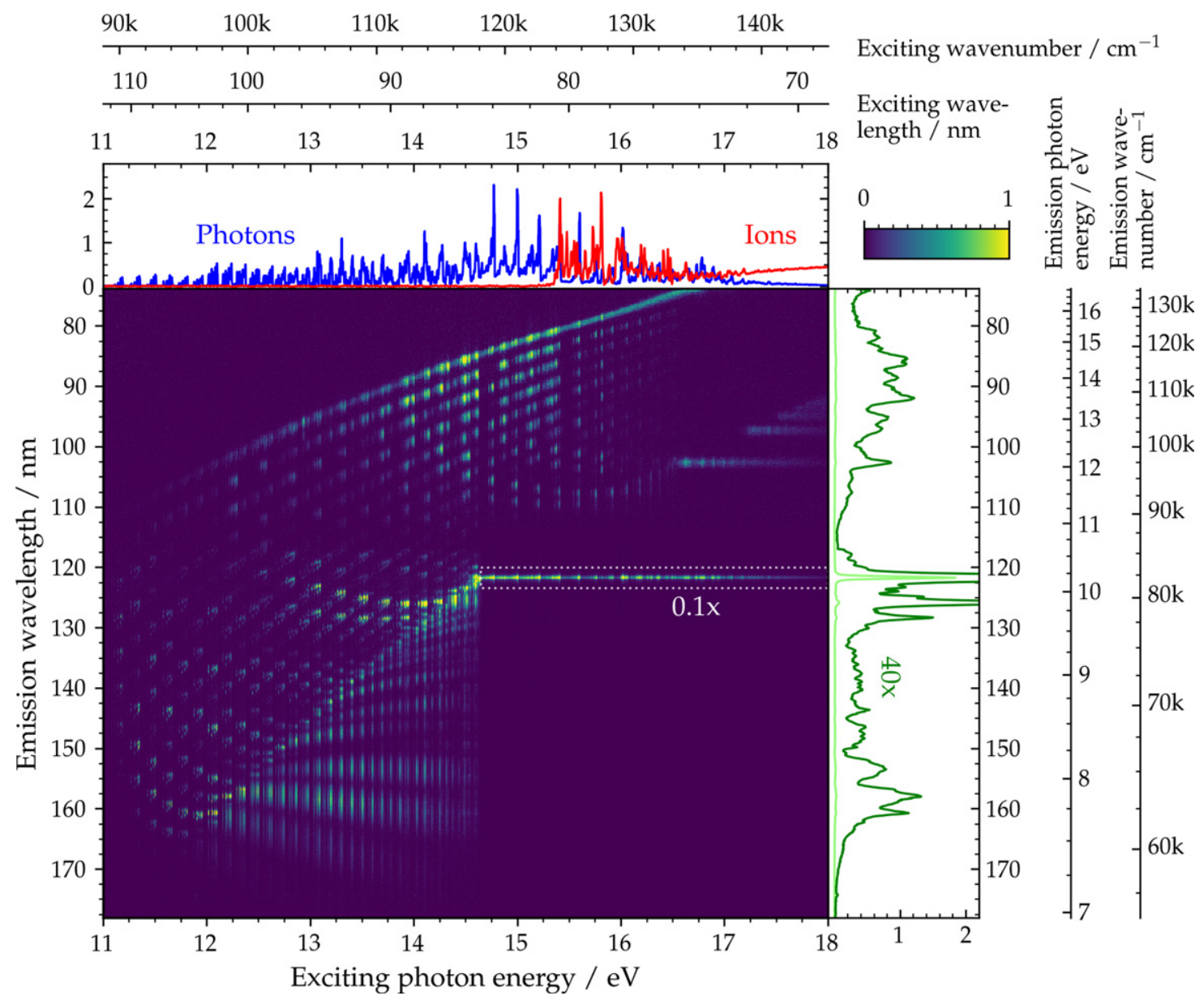

Figure 6. Complete VUV/FUV $\mathrm{H}_{2}$ PhexPhem map with rovibronic resolution in excitation and emission covering all singly excited electronic states. The fluorescence excitation function (intensities integrated over the whole emission wavelength range, blue solid line) and the recorded ionization yield (red) is plotted above the map, while the emission spectrum integrated over the whole exciting-photon energy range (green) is shown to the right. The $\mathrm{Ly}_{\alpha}$-line is reduced in intensity by a factor of 10 before the non-linear intensity scaling [equation (2)] with $a=15$ has been applied.

intensity scale. If required, parts of the map or the whole map relevant for the study of particular aspects can be displayed differently, of course not changing the underlying data.
The sheer number of individual measurements assembled for this single representation demands a particular attention to proper normalization if all features are to be put on the same 
relative intensity scale. The presented measurements, however, did not have this ambition rather than it represents proof of principle experiments trading off quantitative information for a full set of qualitative decay pathway details. Hence, we will not discuss measurement uncertainties, as this will be the task of forthcoming experiments.

\section{Discussion}

As the collected data and representation contains a wealth of information it is necessary to familiarize with its major features, most of which are well known from past work dedicated to a particular aspect of the whole PhexPhem map. To identify these features the present PhexPhem map is compared to a comparatively simple simulation. The calculation is based on solving the non-relativistic nuclear part of the Schrödinger equation in Born-Oppenheimer approximation (Born and Oppenheimer 1927) with $\hat{p}$ the momentum operator, $\mu$ the reduced mass, $J$ the rotational quantum number, $\chi$ the nuclear wavefunction and $E$ the nuclear term energy.

$$
\left(\frac{\hat{p}^{2}}{2 \mu}+U(R)-\frac{\hbar^{2}}{2 \mu R^{2}}(J(J+1))\right) \chi(R)=E \chi(R)
$$

The potential energy curves $U(R)$ for the electronic states have been taken theoretical term values where a variety of corrections have already been considered to increase the accuracy of the Born-Oppenheimer approximation (Dressler and Wolniewicz 1986, Wolniewicz and Dressler 1988, Wolniewicz 1993, 1995a, Staszewska and Wolniewicz 2002). With these, equation (3) is solved numerically by expressing its Hamilton operator as a discrete matrix on a finite grid and solving the resulting eigenvalue problem to obtain eigenvalues $\tilde{E}$ and eigenvectors $\tilde{\chi}$ as numerical approximations of the respective nuclear wavefunctions $\chi$ and energies $E$. The grid is chosen to discretize the internuclear coordinate $R$ between $\left[R_{\min }, R_{\max }\right]$ into $\mathrm{N}$ equidistant points with distances $\mathrm{d} R$. Minimum and maximum internuclear distances considered in the calculations depend on the specific electronic potential. Typically, the minimum value has been chosen to $a_{0} / 2$ and the maximum to equal or larger than $20 \cdot a_{0}$, with $a_{0}$ being the Bohr radius. The eigenvalue problem has been solved by diagonalizing the Hamilton operator in equation (3). For this purpose the kinetic energy part of the Hamilton operator is solved by the Fourier grid Hamiltonian method (Marston and Balint-Kurti 1989). Eigenvalues $\tilde{E}$ and eigenvectors $\tilde{\chi}$ for the final matrix $H$ are determined using the QR-algorithm (Gates and Gragg 1997) for Hermitian matrices in LAPACK (Anderson et al 1999). Each element in the eigensystem represents a specific vibronic level (of a specific electronic state). The resulting set contains both bound vibrational levels up to the dissociation limit and unbound continuum levels beyond it.

Photon emission in the current experiment has been observed as a result of the processes

(a) $\mathrm{H}_{2}\left(q^{\prime \prime}\right)+h \nu_{\text {exc }} \rightarrow \mathrm{H}_{2}\left(q^{\prime}\right) \rightarrow \mathrm{H}_{2}(q)+h \nu_{\text {fluo }}$ (b) $\mathrm{H}_{2}\left(q^{\prime \prime}\right)+h \nu_{\text {exc }} \rightarrow \mathrm{H}_{2}\left(q^{\prime}\right) \rightarrow(\mathrm{H}+\mathrm{H})(q)+h \nu_{\text {fluo }}$ (c) $\mathrm{H}_{2}\left(q^{\prime \prime}\right)+h \nu_{\text {exc }} \rightarrow \mathrm{H}_{2}\left(q^{\prime}\right) \rightarrow \mathrm{H}+\mathrm{H}^{*} \rightarrow \mathrm{H}+\mathrm{H}+h \nu_{\text {fluo }}$.
Among the decay mechanisms involving photon emission the most prominent ones are molecular bands (4(a)), continuum emissions (4(b)), and fragment fluorescence (4(c)). They require slightly different approaches in the simulation.

\subsection{Intensities of molecular bands (bound-bound transitions in emission)}

The intensity profile for a specific fluorescence transition $\Delta E_{\text {fluo }}=E_{q^{\prime}}-E_{q^{\prime \prime}}$ within a molecular band has been calculated by

$$
\begin{aligned}
I\left(h \nu_{\mathrm{exc}}, h \nu_{\text {fluo }}\right)= & \sum_{q} B_{q q^{\prime}} \cdot G\left(h \nu_{\mathrm{exc}}, E_{q^{\prime}}-E_{q}\right) \cdot A_{q^{\prime} q^{\prime \prime}} \\
& \cdot R\left(h \nu_{\text {fluo }}, E_{q^{\prime}}-E_{q^{\prime \prime}}\right)
\end{aligned}
$$

with $G$ and $R$ being Gaussians centred around the excitation energy $E_{q^{\prime}}-E_{q}$ and around the emission energy $E_{q^{\prime}}-E_{q^{\prime \prime}}$, respectively, with half widths $\sigma_{x}=1 /(2 \sqrt{2 \ln 2}) \cdot \Delta E_{x}$ proportional to the experimentally determined full widths at half maximum $\Delta E_{x}$ in excitation and in fluorescence detection. The transition probabilities per unit time are represented by the Einstein coefficients for absorption

$$
B_{q q^{\prime}}=\frac{2}{3} \alpha c^{4} \hbar^{2}\left|\left\langle\psi_{q^{\prime}}|\hat{d}| \psi_{q}\right\rangle\right|^{2}
$$

where the radiation energy volume density has been defined per unit frequency interval, and for spontaneous emission

$$
A_{q^{\prime} q^{\prime \prime}}=\frac{4}{3} \alpha c\left(\frac{E_{q^{\prime}}-E_{q^{\prime \prime}}}{\hbar c}\right)^{3}\left|\left\langle\psi_{q^{\prime \prime}}|\hat{d}| \psi_{q^{\prime}}\right\rangle\right|^{2},
$$

using the dipole matrix elements. In Born-Oppenheimer approximation these are proportional to the product of Hönl-London and Franck-Condon factors weighed with the electronic transition moment

$$
\left|\left\langle\psi_{q^{\prime}}|\hat{d}| \psi_{q}\right\rangle\right|^{2} \propto S_{J J^{\prime}} \cdot\left|\int \chi^{\prime} \chi d_{q q^{\prime}}^{e}(R) \mathrm{d} R\right|^{2} .
$$

The electronic transition moments $d_{q q^{\prime}}^{e}(R)$ contain the dependence of the electronic matrix element on the nuclear coordinate and have been taken from accurate calculations in literature, where available (Wolniewicz and Staszewska 2003a, 2003b). In all other cases the transition moment has been assumed to be constant. Experiments have been performed at room temperature. The initial vibronic state is, therefore, $\mathrm{H}_{2} X^{1} \Sigma_{g}^{+}(\mathrm{v}=0)$ with negligible population of higher vibrational states. Within this state the population distribution of rotational levels is described by a Boltzmann distribution taking into account the nuclear multiplicity and amounts to relative probabilities of $0.13,0.66,0.12$, and 0.09 for $J=0,1,2,3$ and is less than $1 \%$ for all higher rotational levels altogether.

Generally molecular hydrogen in room-temperature populated rovibronic levels of its vibronic ground state is excited by a photon of energy $h \nu_{\text {exc }}$ into a rovibronic level of an electronically excited state $Y(Y=$ all accessible singly excited 
(a)

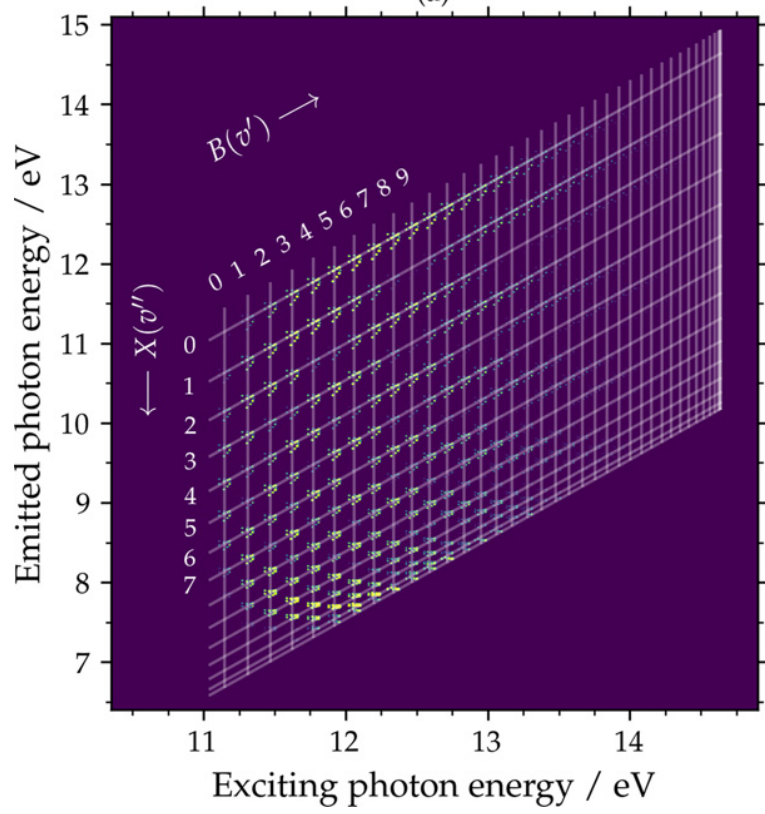

(b)

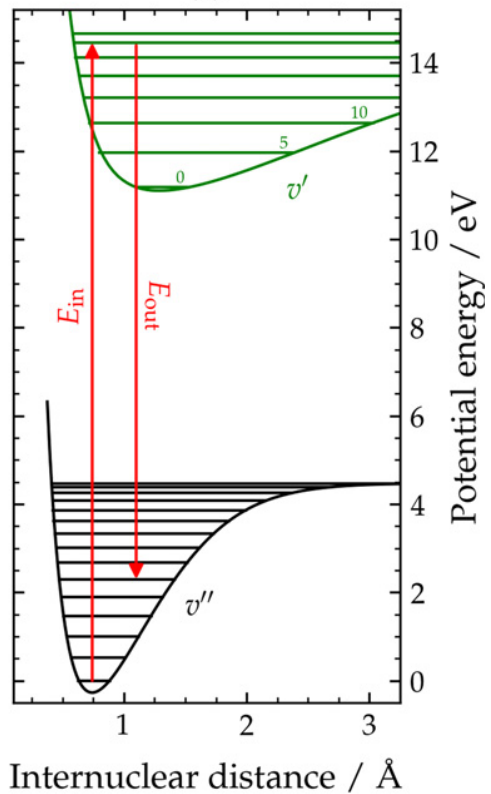

Figure 7. (a) Calculated contributions of molecular bands induced by the processes $\mathrm{H}_{2} X^{1} \Sigma_{g}^{+}\left(\mathrm{v}^{\prime \prime}=0, J^{\prime \prime}\right)+h \nu_{\mathrm{exc}} \rightarrow \mathrm{H}_{2} B^{1} \Sigma_{u}^{+}\left(\mathrm{v}^{\prime}, J^{\prime}\right)$ $\rightarrow \mathrm{H}_{2} X^{1} \Sigma_{g}^{+}\left(\mathrm{v}^{\prime \prime}, J^{\prime \prime}\right)+h \nu_{\text {fluo }}$ (Lyman band system) to the total photon excitation emission map. Rovibronic states $v^{\prime}, J^{\prime}$ are selected by the excitation energy and appear as bands arranged in vertical columns. Bands due to transitions into the same final rovibronic states $v^{\prime \prime}, J^{\prime \prime}$ appear as parallel bisectorial lines. An exemplary process is sketched in the potential energy diagram in panel (b). Processes involving other electronic intermediate states result in similar checkerboard patterns in the map.

(a)

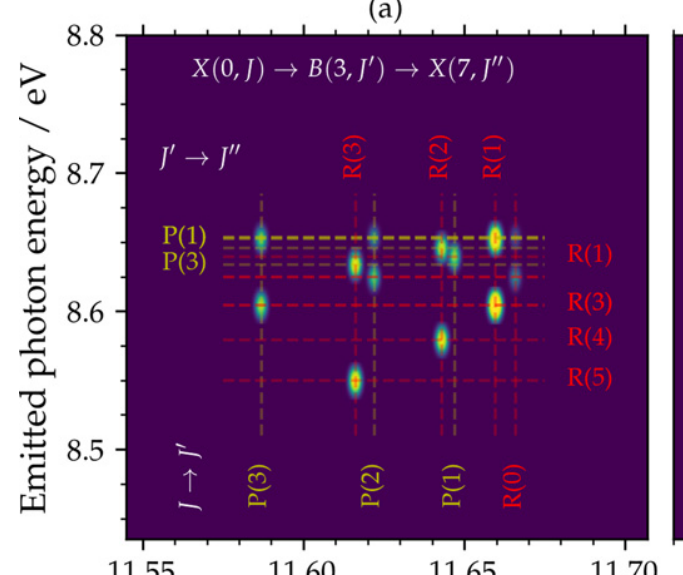

(b)

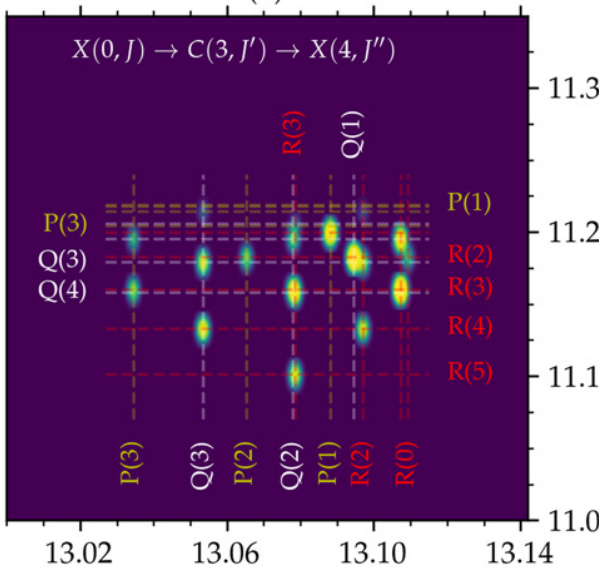

Exciting photon energy / eV

Figure 8. Simulated rotational substructure for two exemplarily chosen bound-bound transition bands. (a) A Lyman band and (b) a Werner band. The rotational branches for the excitation (horizontal scale) and emission (vertical scale) are annotated in yellow for P, white for Q and red for $\mathrm{R}$.

electronic states of $\mathrm{H}_{2}$ ). This level then decays into a number of possible final rovibronic levels of an electronic state $Z$ by emission of corresponding photons of energies $h \nu_{\text {fluo }}$ (or wavelengths $\lambda_{\text {fluo }}$ ). The observed processes are

$$
\begin{aligned}
& \mathrm{H}_{2} X^{1} \Sigma_{g}^{+}\left(\mathrm{v}^{\prime \prime}=0, J^{\prime \prime}\right)+h \nu_{\mathrm{exc}} \\
& \quad \rightarrow \mathrm{H}_{2} Y^{1} \Lambda^{\prime}\left(\mathrm{v}^{\prime}, J^{\prime}\right) \rightarrow \mathrm{H}_{2} Z^{1} \Lambda(\mathrm{v}, J)+h \nu_{\text {fluo }}\left(\text { or } \lambda_{\text {fluo }}\right) .
\end{aligned}
$$

For bound states $Y$ and $Z=X^{1} \Sigma_{g}^{+}\left(\mathrm{v}^{\prime \prime}=0, J^{\prime \prime}\right)$ the wellknown Lyman $(Y=B, Z=X)$ and Werner $(Y=C, Z=X)$ bands as well as bands originating from higher excited electronic states are identified by checkerboard-like features in the PhexPhem maps covering a certain area in the map. Such a feature, representative for the process involving the state $Y=B^{1} \Sigma_{u}^{+}$is shown in figure 7 , where, different as in figure 6, dispersed fluorescence intensities have been plotted as functions of emitted-photon energy rather than emitted-photon wavelength.

The intensity distribution across this checkerboard pattern is a direct portrait of Franck-Condon factors and thus 

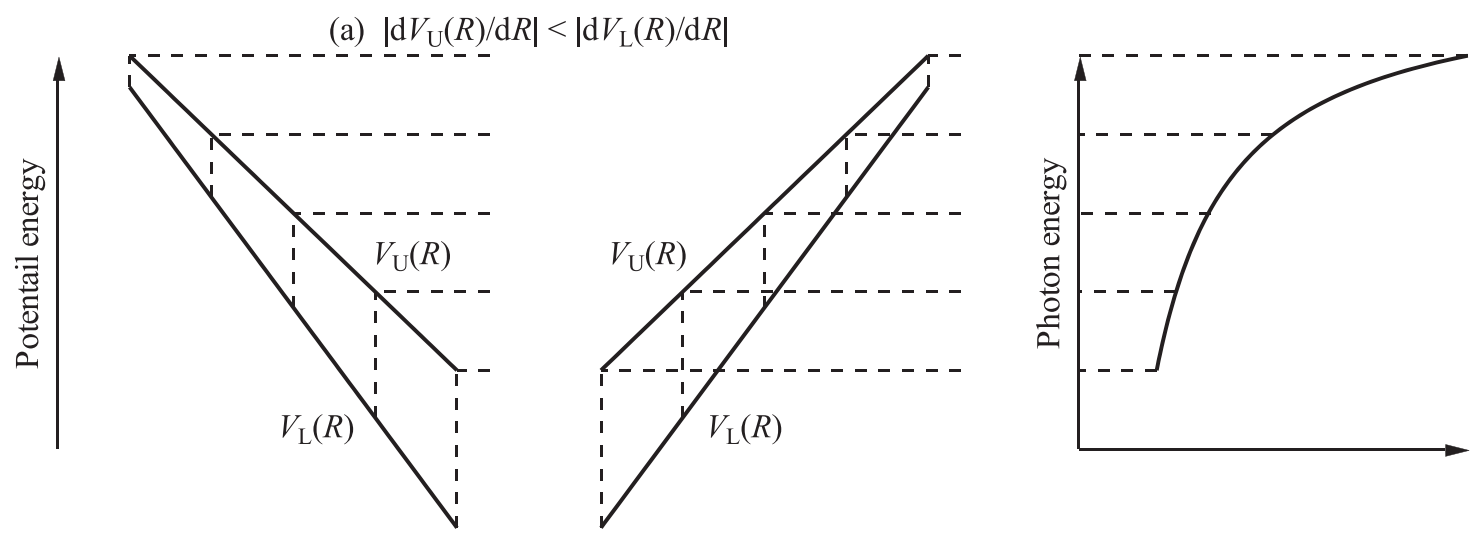

(b) $\left|\mathrm{d} V_{\mathrm{U}}(R) / \mathrm{d} R\right|=\left|\mathrm{d} V_{\mathrm{L}}(R) / \mathrm{d} R\right|$
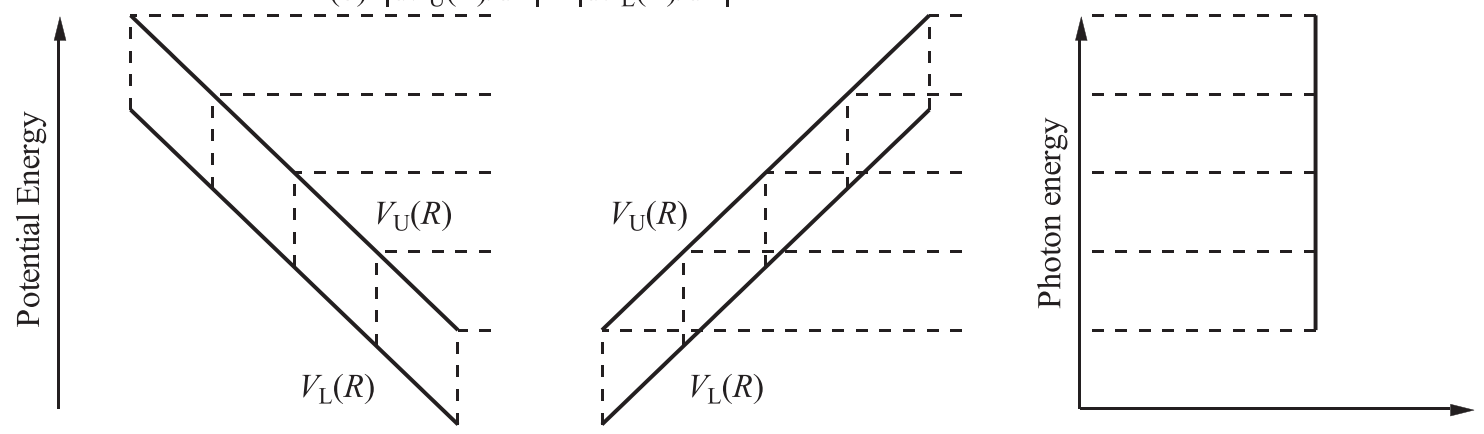

(c) $\left|\mathrm{d} V_{\mathrm{U}}(R) / \mathrm{d} R\right|>\left|\mathrm{d} V_{\mathrm{L}}(R) / \mathrm{d} R\right|$

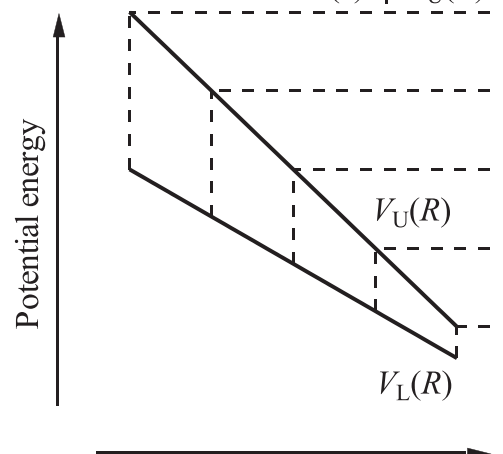

Inner-wall internuclear distance
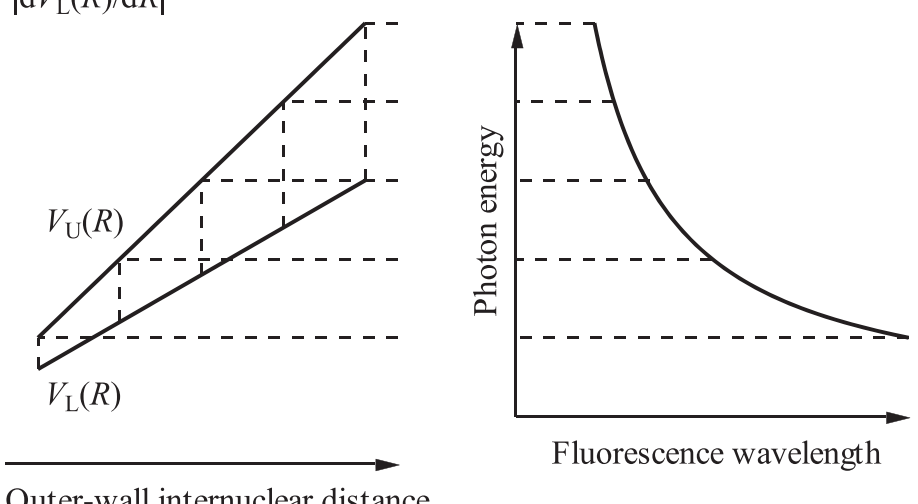

Figure 9. Correspondence scheme for observed band systems when the energy of the exciting-photons are plotted versus the wavelength of the emitted ones. The right hand sides of the three panels (a), (b), (c) are showing the shape of the band systems in the PhexPhem map for the cases that the potential energy derivative with respect to $R$ of the upper state $\mathrm{dV}_{U}(R) / \mathrm{d} R$ is smaller (a), equal (b), or larger (c) as compared to the corresponding derivative $\mathrm{dV}_{L}(R) / \mathrm{d} R$ of the lower state.

a two-dimensional illustration of the vibrational wave function overlaps of the upper and lower states as a function of both vibrational quantum numbers. It visualizes the Condon parabola, which gives the set of lower vibrational levels with the highest intensity for any given upper level in emission. As such, the maximum intensity evolves along those excited levels with a particularly large probability density around the equilibrium distance and those relaxed levels overlapping with the upper turning points. This trend converges strikingly with increasing excitation energy toward the point, where the eventual dissociation of the particular upper state occurs. Depending on the upper electronic state, this corresponds to one half of one or more Condon parabolas. The rotational levels in turn are visible as a substructure in each of the $\left(\mathrm{v}^{\prime}, \mathrm{v}^{\prime \prime}\right)$ features. Figure 8 shows magnified parts of the calculated rotational substructures of two exemplarily chosen bands within our model. While all level combinations form a grid similar to the vibration features, the rotational selection rules reduce the populated nodes significantly. The relative intensities between the different lines corresponding to rovibronic transitions are governed by the Hönl-London factors, which depend on the geometry of the involved electronic states.

A further feature of the PhexPhem map is that it is easy to attain information on the potential energy curves of the involved states. This is schematically shown in figure 9. 
(a)

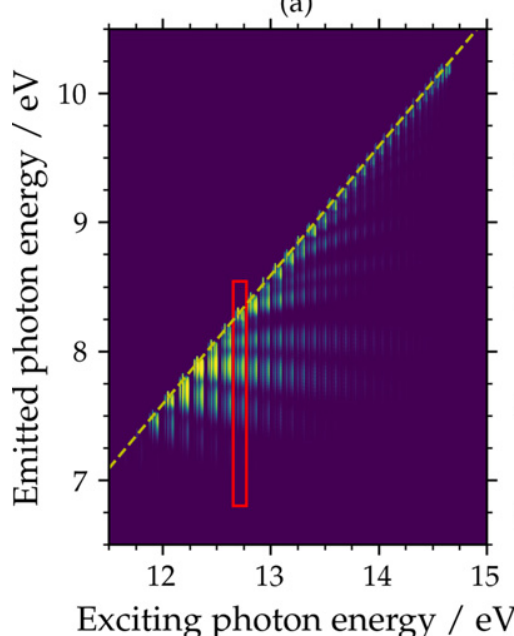

(b)

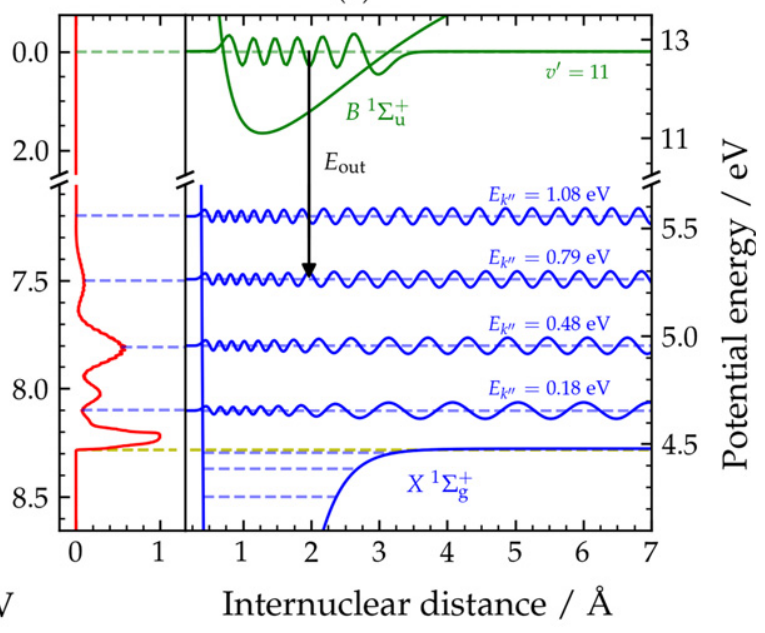

Figure 10. (a) Calculated features in the two-dimensional emission map for the Condon diffraction bands from the $\mathrm{H}_{2} B^{1} \Sigma_{u}^{+}\left(\mathrm{v}^{\prime}, J^{\prime}\right)$ rovibronic states. (b) The calculated spectral distribution due to transitions from the upper bound level $\mathrm{H}_{2} B^{1} \Sigma_{u}^{+}\left(\mathrm{v}^{\prime}=11, J^{\prime}\right)$ (red, corresponds to the intensity recorded in the red boxed area of (a)) and the potential energy diagram. Continuous (dissociative) nuclear wavefunctions of the ground state are plotted for four selected kinetic energies of the dissociation fragments.

The three panels of figure 9 display the cases when the potential energy derivative with respect to $R$ of the upper state $\mathrm{dV}_{U}(R) / \mathrm{d} R$ is smaller (a), equal (b), or larger (c) as compared to the corresponding derivative $\mathrm{dV}_{L}(R) / \mathrm{d} R$ of the lower state. The corresponding shapes of the recorded band systems in a PhexPhem map with exciting-photon energy plotted in energy units and photon emission plotted in wavelength units are shown on the right hand sides of the three panels.

\subsection{Transition intensities to dissociation continua (bound-continuum transitions in emission)}

The hydrogen molecule is capable of radiative transitions into the vibrational continuum of a lower state to form the Condon diffraction bands (Condon 1928), in astrophysics frequently called Solomon process (Stecher and Williams 1967). The corresponding features in the two-dimensional map will now be exemplified for the process

$$
\begin{aligned}
& \mathrm{H}_{2} X^{1} \Sigma_{g}^{+}\left(\mathrm{v}^{\prime \prime}=0, J^{\prime \prime}\right)+h \nu_{\text {exc }} \\
& \quad \rightarrow \mathrm{H}_{2} B^{1} \Sigma_{u}^{+}\left(\mathrm{v}^{\prime}, J^{\prime}\right) \rightarrow \mathrm{H}_{2} X^{1} \Sigma_{g}^{+}\left(\mathrm{v}>\mathrm{v}_{\text {limit }}, J\right)+h \nu_{\text {fluo }} .
\end{aligned}
$$

For practical purposes the discretized continuum levels $v$ have been restricted in the calculations to levels somewhat close to the dissociation limit, where the emission intensity will not be negligibly small, in the present case to $v_{\text {limit }} \leqslant v \leqslant 135$. Describing continuum levels by discrete solutions requires that the energetic spacings between these solutions are smaller than the detector resolution.

While fluorescence transitions from a bound to a dissociating continuum state is generally possible for all electronically excited states, this process is particularly prominent for transitions from the $\mathrm{H}_{2} B^{1} \Sigma_{u}^{+}\left(\mathrm{v}^{\prime}, J^{\prime}\right)$ states due to the rather wide potential energy curve (Wang et al 2011). It allows a significant probability density at larger internuclear distances, where no suitable bound level of the ground state exists. The generated spectral distribution is continuous with characteristic variations in intensity (Dalgarno et al 1970). These are caused by the slow variation of the continuum wavefunctions due to the varying energy difference to the dissociation limit. This in turn causes a slow variation of the overlap between the continuum wavefunctions and the wavefunction of the upper bound state. The calculated characteristic features of Condon diffraction bands in the two-dimensional map for the $\mathrm{H}_{2} B^{1} \Sigma_{u}^{+}\left(\mathrm{v}^{\prime}, J^{\prime}\right)$ states is shown in figure 10 together with the corresponding potential energy diagram.

\subsection{Intensities of transitions of excited atomic fragments}

An excitation from the ground state into the vibrational continuum of an electronic state will dissociate the molecule into two hydrogen atoms. The electronic excitation of the initial molecular state (in the present case of a single electron) will be transferred to an electronic excitation of one of the fragments, which will then return to its atomic ground state by photon emission. This process can be summarized by:

$$
\begin{gathered}
\mathrm{H}_{2} X^{1} \Sigma_{g}^{+}\left(\mathrm{v}^{\prime}=0, J^{\prime \prime}\right)+h \nu_{\mathrm{exc}} \rightarrow \mathrm{H}_{2} Y^{1} \Lambda^{\prime}\left(\mathrm{v}^{\prime} \geqslant \mathrm{v}^{\prime}{ }_{\text {limit }}, J^{\prime}\right) \\
\rightarrow \mathrm{H}(n l)+\mathrm{H}(1 s) \rightarrow \mathrm{H}(1 s)+\mathrm{H}(1 s)+h \nu_{\text {fluo }} .
\end{gathered}
$$

The transition into the dissociating molecular state $Y$ can still be modelled by equation (7). Assuming the population to be identical to that of the forming $\mathrm{H}(n l)$ atoms, their emissions may be obtained using the respective atomic branching ratios. As for the calculations of the previously discussed continuum emissions, the number of upper discretized continuum levels $\mathrm{v}^{\prime}$ have been limited to $\mathrm{v}^{\prime} \approx 1000$ corresponding to $E_{\mathrm{v}^{\prime}} \approx 18 \mathrm{eV}$. Also here the spacing of the discrete vibrational continuum levels must be smaller than the bandwidth of the exciting radiation. The corresponding calculated features for the twodimensional representation together with the potential energy curves are shown in figure 11

The calculated process considers emissions of atomic fragments only after direct excitation into the dissociation 
(a)

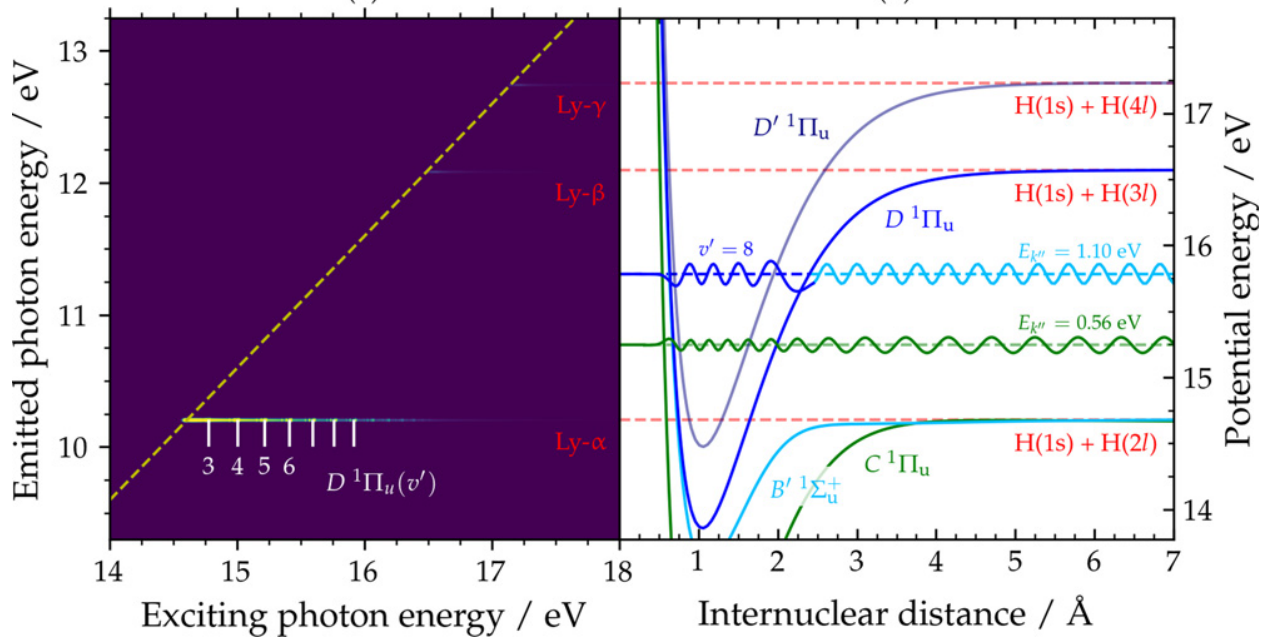

Figure 11. (a) Simulated fluorescence from excited atomic fragments after dissociation. (b) Excited electronic states in the potential energy diagram leading for large internuclear distances to different dissociation continua (red). The excitation can either occur directly into the vibrational continuum or by predissociation.

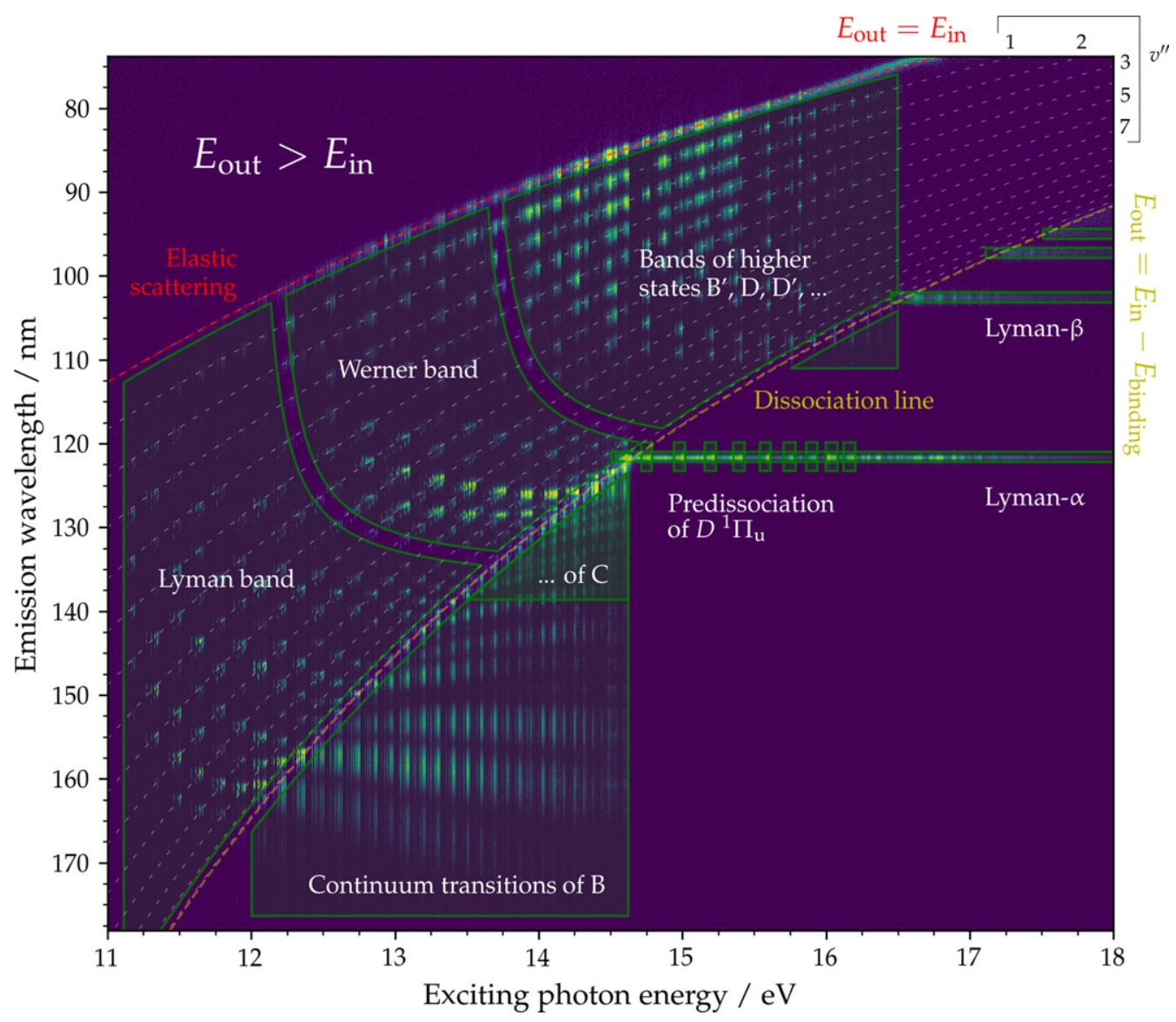

Figure 12. VUV/FUV experimental PhexPhem map of $\mathrm{H}_{2}$ with major features indicated.

continuum. The excitation function for the Lyman series of molecular hydrogen, however, exhibits a wide range of resonances attributed to the predissociation of vibrational levels of higher electronic states through direct (e.g. Fano (1935), (1961)) or indirect coupling (Glass Maujean 1979) with the continuum (Liu et al 2012). The purely adiabatic ansatz of the present model does not allow to describe such processes. But as the predissociation yields of most states in the present experimental range are well known (Guyon et al 1979), they have been approximated by treating the excitation into the predissociating levels partially as a dissociation with subsequent photon emission from the fragments. The same applies 
for autoionization processes beyond the ionization threshold of $15.43 \mathrm{eV}$, because the $\mathrm{H}_{2}$ molecule and its isotopologues possess the rare property to have several vibrational levels in this energy region that are stable against this process and still emit fluorescence. The competition between these decay mechanisms has been investigated for the majority of states (Glass-Maujean et al 2010a, 2010b) and found to selectively quench emissions.

The described calculations are essential for the identification of the major features of the two-dimensional PhexPhem map. The result is shown in figure 12, displaying the experimental data. Clearly practically all band systems emitting in the VUV/ FUV spectral range have been identified. Continuous emissions from the $B, C$, and $D$ states are obvious as well as several Lyman lines of excited fragments. The Lyman lines show characteristic variations in intensities due to predissociation processes.

\section{Conclusion}

We have shown experiments as a proof of concept where dispersed photon emission has been recorded after photon excitation of all singly excited states of $\mathrm{H}_{2}$ as a function of the exciting-photon energy, covering the complete VUV/FUV emission spectral range. About 20000 individual spectra have been appended to represent the emission in a two-dimensional PhexPhem map. In the PhexPhem map molecular processes like dipole-allowed rovibronic transitions between bound states, transitions between a bound state and a dissociation continuum, predissociation, and ionization are visible at the same time, facilitating the identification of particular features. This representation of the data has the potential to be included in standard molecular physics textbooks to explain theoretical concepts of molecular physics like the Franck-Condon principle, potential energy curves, and molecular state interactions. In future experiments a quantitative intensity determination for all observed features will be possible on a joint intensity scale.

\section{Acknowledgments}

The staff of BESSY II is gratefully acknowledged for hands-on help and support during the beamtimes, particularly for keeping the $10 \mathrm{~m}$ NIM beamline in excellent shape. PS gratefully acknowledges a scholarship by the Helmholtz International Centre for FAIR (facility for antiproton and ion research).

\section{ORCID iDs}

Andre Knie (D) https://orcid.org/0000-0002-2208-8838 Andreas Hans (D) https://orcid.org/0000-0002-4176-4766 Arno Ehresmann (1D https://orcid.org/0000-0002-0981-2289

\section{References}

Abgrall H, Roueff E, Launay F, Roncin J Y and Subtil J L 1993 J. Mol. Spectrosc. 157512

Anderson E et al 1999 LAPACK Users' Guide 3rd edn (Philadelphia, PA: Society for Industrial and Applied Mathematics)

Bahrdt J, Frentrup W, Gaupp A, Scheer M, Gudat W, Ingold G and Sasaki S 2001 Nucl. Instrum. Methods Phys. Res. A 467-468 130

Baumgaertel P and Packe I 2016 J. Large-Scale Res. Fac. 2 A53

Born M and Oppenheimer R 1927 Ann. Phys. 8430

Carruthers G R 1970 Astrophys. J. 161 L81

Condon E U 1928 Phys. Rev. 32858

Dalgarno A, Herzberg G and Stephens T L 1970 Astrophys. J. 162 L49

Dressler K and Wolniewicz L 1986 J. Chem. Phys. 852821

Ehresmann A, Schmoranzer H, Vollweiler F, Mentzel G and Schartner K-H 1994b Phys. Lett. A 184 432-4

Ehresmann A, Vollweiler F, Schmoranzer H, Sukhorukov V L, Lagutin B M, Petrov I D, Mentzel G and Schartner K-H 1994a J. Phys. B: At. Mol. Opt. Phys. 27 1489-96

Fano U 1935 Nuovo Cim. 12154

Fano U 1961 Phys. Rev. 1241866

Gates K and Gragg W B 1997 J. Comput. Appl. Math. 86195

Glass-Maujean M 1979 Chem. Phys. Lett. 68320

Glass-Maujean M, Jungen C, Reichardt G, Balzer A, Schmoranzer H, Ehresmann A, Haar I and Reiss P 2010b Phys. Rev. A 82 062511

Glass-Maujean M, Jungen C, Schmoranzer H, Knie A, Haar I, Hentges R, Kielich W, Jänkälä K and Ehresmann A 2010a Phys. Rev. Lett. 104183002

Glass-Maujean M, Jungen C, Schmoranzer H, Tulin I, Knie A, Reiss P and Ehresmann A 2013 J. Mol. Spectrosc. 293-294 19

Glass-Maujean M, Jungen C, Vasserot A M, Schmoranzer H, Knie A, Kübler S, Ehresmann A and Ubachs W 2017 J. Mol. Spectrosc. 33822

Guyon P M, Breton J and Glass-Maujean M 1979 Chem. Phys. Lett. 68314

Hans A et al 2015 Phys. Rev. A 92032511

Hans A, Schmidt P, Ozga C, Hartmann G, Holzapfel X, Ehresmann A and Knie A 2018 Materials 11869

Herzberg G and Jungen C 1972 J. Mol. Spectrosc. 41425

Liu X, Shemansky D E, Johnson P V, Malone C P, Khakoo M A and Kanik I 2012 J. Phys. B: At. Mol. Opt. Phys. 45015201

Marston C C and Balint-Kurti G G 1989 J. Chem. Phys. 913571

Meyer D M, Lauroesch J T, Sofia U J, Draine B T and Bertoldi F 2001 Astrophys. J. $\mathbf{5 5 3}$ L59

Moos H W et al 2010 Astrophys. J. 538 L1

Morton D C and Dinerstein H L 1976 Astrophys. J. 2041

Reichardt G et al 2001 Nucl. Instrum. Methods Phys. Res. A 467-468 462

Salumbides E J, Bagdonaite J, Abgrall H, Roueff E and Ubachs W 2015 Mon. Not. R. Astron. Soc. 450 1237-45

Schmoranzer H, Liebel H, Vollweiler F, Müller-Albrecht R, Ehresmann A, Schartner K-H and Zimmermann B 2001 Nucl. Instrum. Methods Phys. Res. A 467-468 1526-8

Schmoranzer H, Molter K, Noll T and Imschweiler J 1986 Nucl. Instrum. Methods Phys. Res. A 246 485-7

Spitzer L J, Cochran W D and Hirshfeld A 1974 Astrophys. J. Suppl. Ser. 28373

Staszewska G and Wolniewicz L 2002 J. Mol. Spectrosc. 212208

Stecher T P and Williams D A 1967 Astrophys. J. Lett. 14929 
Takezawa S 1970 J. Chem. Phys. 522575

Ubachs W 2018 Space Sci. Rev. 2143

Ubachs W, Bagdonaite J, Salumbides E J, Murphy M T and Kaper L 2016 Rev. Mod. Phys. 88021003

Ubachs W, Salumbides E J, Murphy M T, Abgrall H and Roueff E 2019 Astron. Astrophys. 622 A127

Wakelam V et al 2017 Mol. Astrophys. 91

Wang J, Kim K S and Baerends E J 2011 J. Chem. Phys. 135 074111

Wolniewicz L 1993 J. Chem. Phys. 991851
Wolniewicz L 1995a J. Chem. Phys. 1031792

Wolniewicz L and Dressler K 1988 J. Chem. Phys. 883861

Wolniewicz L and Staszewska G 2003a J. Mol. Spectrosc. 217 181

Wolniewicz L and Staszewska G 2003b J. Mol. Spectrosc. 22045

Xiong T, Xu Y-c., Yang K, Hiraoka N and Zhu L-F 2019 Astrophys. J. 885163

Zimmermann B, Schartner K-H, Wilhelmi O, Kammer S, Liebel H, Ehresmann A and Schmoranzer H 2004 J. Phys. B: At. Mol. Opt. Phys. 37 511-23 